\title{
Chaos for finitely generated semigroup actions
}

\author{
Lidong Wanga, ${ }^{\mathrm{a},}$, Yingcui Zhao ${ }^{\mathrm{a}}$, Zhenyan Chu $\mathrm{u}^{\mathrm{b}, *}$ \\ a School of Mathematical Sciences, Dalian University of Technology, Dalian, 116024, People's Republic of China. \\ ${ }^{b}$ Department of Mathematics, Dalian Minzu University, Dalian, 116600, People's Republic of China.
}

Communicated by X. Z. Liu

\begin{abstract}
In this paper, we define and study Li-Yorke chaos and distributional chaos along a sequence for finitely generated semigroup actions. Let $X$ be a compact space with metric $d$ and $G$ be a semigroup generated by $f_{1}, f_{2}, \cdots f_{m}$ which are finitely many continuous mappings from $X$ to itself. Then we show if $(X, G)$ is transitive and there exists a common fixed point for all the above mappings, then $(X, G)$ is chaotic in the sense of Li-Yorke. And we give a sufficient condition for $(X, G)$ to be uniformly distributionally chaotic along a sequence and chaotic in the strong sense of Li-Yorke. At the end of this paper, an example on the one-sided symbolic dynamical system for $(X, G)$ to be chaotic in the strong sense of Li-Yorke and uniformly distributionally chaotic along a sequence is given. (C)2017 All rights reserved.
\end{abstract}

Keywords: Li-Yorke chaos, distributional chaos along a sequence, finitely generated semigroup actions. 2010 MSC: 54H20, 22B99, 37B05.

\section{Introduction}

In general, a dynamical system, is understood as a pair $(X, f)$, where $X$ is a space with a metric $d$ and $f$ is a continuous mapping from $X$ to itself. This term is used in the case where it is necessary to study the dynamics of transformations of the space $X$ caused by the multiple application of the function $f$. Note that the functions formed by the repeated application of $f$ are elements of the semigroup $F=\left\{f^{n} \mid n \in Z, n \geqslant 0\right\}$ with the operation of composition $f^{m} \circ f^{n}=f^{m+n}$. This viewpoint suggests the following generalizations:

Consider an arbitrary semigroup $G$. We associate each $g \in G$ with a continuous function $f_{g}: X \rightarrow X$. Moreover, for any $g, h \in G$, the equality $f_{g \circ h}=f_{g}\left(f_{h}\right)$ must be true. If $G$ contains the identity element $e$, then the condition $f_{e}(x)=x$ must also be satisfied. In this case, we can say that the semigroup $G$ acts on $X$.

Further, instead of $f_{g}(x)$, we write $g(x)$. For any $g_{1}, g_{2} \in G$, we can write $g_{1}, g_{2}$ instead of $g_{1} \circ g_{2}$. Note that, for different elements $g, h \in G$, their actions (i.e., the corresponding functions $f_{g}$ and $f_{h}$ ) may coincide.

\footnotetext{
*Corresponding author

Email addresses: wld0707@126.com (Lidong Wang), zycchaos@126.com (Yingcui Zhao), chuzhenyan8@163.com (Zhenyan Chu)
}

doi:10.22436/jnsa.010.07.38 
More and more scholars study series of properties for semigroup actions. Kontoprova and Megrelishvili [2] studied sensitivity of semigroup actions. Polo [3] studied sensitive dependence on initial conditions and chaotic group actions. In order to study the topological transitivity and mixing for group actions, Gairns et al. introduced the notions of asymptotic, proximal, transitive of group in [1] respectively as follows:

Definition 1.1. For given $\varepsilon$, a pair $(x, y)$ is called $\varepsilon$-asymptotic if there are at most finitely many elements $g \in \mathrm{G}$ for which

$$
\mathrm{d}(\mathrm{g}(\mathrm{x}), \mathrm{g}(\mathrm{y}))>\varepsilon
$$

The pair $(x, y)$ is called asymptotic if it is $\varepsilon$-asymptotic for any $\varepsilon>0$.

Definition 1.2. A pair $(x, y)$ is called proximal, if for any $\delta>0$, there exists an element $g \in G$ for which $\mathrm{d}(\mathrm{g}(\mathrm{x}), \mathrm{g}(\mathrm{y}))<\delta$.

Definition 1.3. A system $(X, G)$ is called transitive, if for any open nonempty sets $U, V \subset X$, there exists $g \in G$ such that $g(U) \cap V \neq \varnothing$. We say that a point $x$ is transitive if its orbit $\{g(x) \mid g \in G\}$ is dense in $X$.

In addition, since Wang first gave the definition of sequentially distributional chaos in [6], the research of it has had a great influence on topology (see [5, 7-16]). However, the notion about sequentially distributional chaos for group actions has not been given so far. On this basis, we define Li-Yorke chaos and distributional chaos along a sequence for the finitely generated semigroup actions, and show if $(X, G)$ is transitive and there exists a fixed point for all the above mappings, then $(X, G)$ is chaotic in the sense of Li-Yorke on the basis of [17]. And we give a sufficient condition for $(X, G)$ to be uniformly distributionally chaotic along a sequence and chaotic in the strong sense of Li-Yorke on the basis of [5]. At the end of this paper, an example on the one-sided symbolic dynamical system for $(X, G)$ to be chaotic in the strong sense of Li-Yorke and uniformly distributionally chaotic along a sequence is given.

\section{Preliminaries and basic concepts}

In this paper, we always suppose that $X$ is a compact space with metric $d$ and $F=\left\{f_{1}, f_{2}, \cdots, f_{m}\right\}$ is a $m$-tuple of continuous maps from $X$ to itself. For any $n>0$, put

$$
F^{n}=\left\{f_{i_{1}} \circ f_{i_{2}} \circ \cdots \circ f_{i_{n}} \mid i_{1}, i_{2}, \cdots, i_{n}=1 \text { or } 2 \text { or } \cdots \text { or } m\right\} .
$$

Let $G=\bigcup_{n=1}^{\infty} F^{n}$, then it is easy to check $G$ is a semigroup generated by $f_{1}, f_{2}, \cdots f_{m}$.

Then, we introduce some basic concepts.

Definition 2.1. The real-valued function $g: X \rightarrow[0,+\infty]$ is called to be semicontinuous, if for any a $\in$ $[0,+\infty], g^{-1}([a,+\infty])$ is a closed set of $X$.

Definition 2.2. A system $(X, G)$ is chaotic in the strong sense of Li-Yorke if there exists an uncountable set $S$ satisfying there exists $\varepsilon>0$ such that for any distinct $x, y \in S,(x, y)$ is a Li-Yorke $\varepsilon$-pair, i.e., it is proximal but not $\varepsilon$-asymptotic.

Definition 2.3. A pair $(x, y)$ is called a Li-Yorke pair if it is proximal but not asymptotic. A system $(X, G)$ is called to be chaotic in the sense of Li-Yorke, if there is an uncountable set $S \subset X$ such that for any distinct points $x, y \in S,(x, y)$ is a Li-Yorke pair.

For any $i>0$ put

$$
\begin{gathered}
T^{(i)}=\left\{\tau^{(i)}=t_{1} t_{2} \cdots t_{i} \mid t_{j}=1 \text { or } 2 \text { or } \cdots \text { or } m, j=1,2, \cdots, i\right\}, \\
g_{\tau^{(i)}}=f_{t_{1}} \circ f_{t_{2}} \circ \cdots \circ f_{t_{i}} .
\end{gathered}
$$


Definition 2.4. A system $(X, G)$ is called to be distributionally chaotic if there exists an uncountable set $S \subset X$ satisfying for any distinct points $x, y \in S,(x, y)$ is a distributionally chaotic pair, i.e.,

(1) $\liminf _{n \rightarrow \infty} \frac{\sum_{i=1}^{n} \sum_{\tau^{(i)} \in T^{(i)}} x_{[0, \delta)}\left(d\left(g_{\tau^{(i)}}(x), g_{\tau^{(i)}}(y)\right)\right)}{\sum_{i=1}^{n} m^{i}}=0$ for some $\delta>0$;

(2) $\limsup _{n \rightarrow \infty} \frac{\sum_{i=1}^{n} \sum_{\tau^{(i)} \in T^{(i)}} x_{[0, \varepsilon)}\left(d\left(g_{\tau^{(i)}}(x), g_{\tau^{(i)}}(y)\right)\right)}{\sum_{i=1}^{n} m^{i}}=1$ for any $\varepsilon>0$.

Definition 2.5. A system $(X, G)$ is called to be distributionally chaotic along a sequence $\left\{p_{i}\right\}$ of positive integers if there exists an uncountable set $S \subset X$ satisfying for any distinct points $x, y \in S,(x, y)$ is a distributionally chaotic pair along a sequence, i.e.

(1) $\liminf _{n \rightarrow \infty} \frac{\sum_{i=1}^{n} \sum_{\tau^{\left(p_{i}\right)} \in T^{\left(p_{i}\right)}} x_{[0, \delta)}\left(d\left(g_{\tau}\left(\mathfrak{p}_{i}\right)\right.\right.}{\sum_{i=1}^{n} m^{p_{i}}}=0$ for some $\delta>0$;

(2) $\limsup _{n \rightarrow \infty} \frac{\sum_{i=1}^{n} \sum_{\left.\tau_{\left(\mathfrak{p}_{i}\right.}\right)_{\in T}\left(\mathfrak{p}_{\mathfrak{i}}\right)} x_{[0, \varepsilon)}\left(\mathrm{d}\left(\mathrm{g}_{\left.\tau^{(} \mathfrak{p}_{\mathfrak{i}}\right)}(\mathrm{x}), g_{\tau}\left(\mathfrak{p}_{\mathfrak{i}}\right)(y)\right)\right)}{\sum_{i=1}^{n} m^{p_{i}}}=1$ for any $\varepsilon>0$.

Definition 2.6. Let system $(X, G)$ be distributionally chaotic along a sequence $\left\{p_{i}\right\}$. The distributionally Chaos along a sequence is called to be uniform if there exists $\delta>0$ such that for any distinct points $x, y \in S$,

$$
\liminf _{n \rightarrow \infty} \frac{\sum_{i=1}^{n} \sum_{\tau^{\left(p_{i}\right)} \in T^{\left(p_{i}\right)}} x_{[0, \delta)}\left(d\left(g_{\tau^{\left(p_{i}\right.}}(x), g_{\tau^{\left(p_{i}\right)}}(y)\right)\right)}{\sum_{i=1}^{n} m^{p_{i}}}=0
$$

Definition 2.7. There exists an increasing sequence of positive integers $\left\{p_{i}\right\}$. Let

$A R\left(F,\left\{p_{i}\right\}\right)=\{(x, y) \in X \times X \mid$ for any $\varepsilon>0$, there are at most finitely many elements

$$
\left.g \in \bigcup_{m=0}^{\infty} F^{p_{i}} \text { such that } d(g(x), g(y)) \geqslant \varepsilon\right\}
$$

and call it the asymptotic relation with respect to $\left\{p_{i}\right\}$. Let

$$
\operatorname{PR}\left(F,\left\{p_{i}\right\}\right)=\left\{(x, y) \in X \times X \mid \text { for any } \delta>0, \text { there exists } g \in \bigcup_{m=0}^{\infty} F^{p_{i}} \text { such that } d(g(x), g(y))<\delta\right\},
$$

and call it the proximal relation with respect to $\left\{p_{i}\right\}$. Let

$$
\operatorname{DR}\left(F,\left\{p_{i}\right\}\right)=X \times X-P R\left(F,\left\{p_{i}\right\}\right),
$$

and call it the distal relation with respect to $\left\{p_{i}\right\}$. Let

$\operatorname{DCR}\left(F,\left\{p_{i}\right\}\right)=\left\{(x, y) \in X \times X \mid(x, y)\right.$ is a distributionally chaotic pair along a sequence $\left.\left\{p_{i}\right\}\right\}$, and call it the distributionally chaotic relation with respect to $\left\{p_{i}\right\}$.

Remark 2.8. One can see when the semigroup $G$ is generated only by one continuous map $f$, then Definitions 2.2-2.7 are the same with the classical corresponding definitions.

By Definitions 2.2-2.3 and 2.5-2.6, it is easy to know:

Proposition 2.9. If $(\mathrm{X}, \mathrm{G})$ is distributionally chaotic along a sequence, then it is chaotic in the sense of Li-Yorke.

Proposition 2.10. If $(\mathrm{X}, \mathrm{G})$ is uniformly distributionally chaotic along a sequence, then it is chaotic in the strong sense of Li-Yorke. 


\section{Main results and proofs}

Before presenting the first main result, we list several lemmas and propositions which play roles in the proof of the result.

Lemma 3.1 ([4]). If $\mathrm{X}$ is a compact space and $(\mathrm{X}, \mathrm{G})$ is a transitive system, then the set of its transitive points is dense in $\mathrm{X}$.

Lemma 3.2 ([17]). Let $\mathrm{H}$ be a family of real-valued functions from $\mathrm{X}$ to $[0,+\infty]$. Denote $\mathrm{g}: \mathrm{X} \rightarrow[0,+\infty]$ for any $x \in X$,

$$
g(x)=\inf \{h(x) \mid h \in H\} .
$$

If each $\mathrm{h} \in \mathrm{H}$ is semicontinuous, then $\mathrm{g}$ is semicontinuous.

Lemma 3.3 ([17]). Suppose that for each integer $i \geqslant 1, h_{i}: X \rightarrow[0,+\infty]$ is semicontinuous. Let $a \in[0,+\infty]$,

$$
g(x)=\liminf _{i \rightarrow \infty} h_{i}(x), \quad x \in X .
$$

If the set $\mathrm{g}^{-1}([0, \mathrm{a}])$ is dense in $\mathrm{X}$, then it must be a dense $\mathrm{G}_{\delta}$ set of $\mathrm{X}$.

Lemma 3.4. If there exists a transitive point of $(X, G)$ and $\bigcap_{i=1}^{m} F i x\left(f_{i}\right) \neq \varnothing$, in which Fix(f) denotes the set of fixed points of $\mathrm{f}$, then there exists a dense $\mathrm{G}_{\delta}$ set $\mathrm{B}$ in $\mathrm{X} \times \mathrm{X}$ satisfying for any $(\mathrm{x}, \mathrm{y}) \in \mathrm{B}$ and any $\delta>0$,

$$
\exists g \in G, \quad d(g(x), g(y))<\delta .
$$

Proof. Let $\omega$ be the transitive point of $(X, G)$, then $\overline{\operatorname{orb}(\omega)}=X$. For any $z=\left(z_{1}, z_{2}\right) \in \operatorname{orb}(\omega) \times \operatorname{orb}(\omega)$, there exist $\varphi_{1}, \varphi_{2} \in \mathrm{G}$ such that $z_{1}=\varphi_{1}(\omega), z_{2}=\varphi_{2}(\omega)$. Let $v \in \bigcap_{i=1}^{m} \operatorname{Fix}\left(f_{i}\right) \neq \varnothing$. By $\overline{\operatorname{orb}(\omega)}=X$, there exists $\left\{g_{n_{j}}\right\}_{i=1}^{\infty}$ in which $g_{n_{j}} \in F_{j}^{n}$ such that as $n_{j} \rightarrow \infty, g_{n_{j}}(\omega) \rightarrow v$. Thus,

$$
\lim _{j \rightarrow \infty} g_{n_{j}}\left(z_{i}\right)=\lim _{j \rightarrow \infty} g_{n_{j}} \varphi_{i}(\omega)=\varphi_{i}\left(\lim _{j \rightarrow \infty} g_{n_{j}}(\omega)\right)=\varphi_{i}(v)=v, \quad i=1,2 .
$$

Choose $\left\{g_{n}\right\}_{\mathfrak{n}=1}^{\infty}$ such that for any $n \geqslant 1, g_{n} \in F^{n}$ and $\left\{g_{n_{j}}\right\}_{j=1}^{\infty} \subset\left\{g_{n}\right\}_{n=1}^{\infty}$. So,

$$
\liminf _{n \rightarrow \infty} d\left(g_{n}\left(z_{1}\right), g_{n}\left(z_{2}\right)\right) \text {. }
$$

Let $\tilde{F}\left(z_{1}, z_{2}\right)=\liminf _{n \rightarrow \infty} d\left(g_{n}\left(z_{1}\right), g_{n}\left(z_{2}\right)\right)$. Note that $\operatorname{orb}(\omega) \times \operatorname{orb}(\omega)$ is dense in $X \times X$. Then by Lemma 3.3 we can easily know $\tilde{F}^{-1}(0)$ is a dense $G_{\delta}$ set in $X \times X$.

Set $\operatorname{Re}(G)=\left\{x \in X \mid \exists\left\{g_{n_{i}}\right\}_{i=1}^{\infty}, g_{n_{i}} \in F^{n_{i}}, i \geqslant 1\right.$, such that $\left.\lim _{i \rightarrow \infty} g_{n_{i}}(x)=x\right\}$.

Lemma 3.5. If $\overline{\operatorname{Re}(\mathrm{G})=\mathrm{X}}$, then $\operatorname{Re}(\mathrm{G})$ is a dense $\mathrm{G}_{\delta}$-set.

Proof. For any $x \in \operatorname{Re}(\mathrm{G})$, we have

$$
\exists\left\{g_{n}\right\}_{n=1}^{\infty}, \quad g_{n} \in F^{n}, \quad n \geqslant 1 \text { and } n_{i} \text { such that } \lim _{i \rightarrow \infty} g_{n_{i}}(x)=x .
$$

Let $\tilde{\mathrm{F}}: X \rightarrow[0,+\infty)$ and for any $x \in X$,

$$
\tilde{F}(x)=\liminf _{n \rightarrow \infty} \inf _{g \in F^{n}} d(g(x), x) .
$$

It is easy to know $x \in \operatorname{Re}(G)$ if and only if $\tilde{F}(x)=0$. Hence, $\tilde{F}^{-1}(0)$ is dense in $X$.

For each $n \geqslant 1$ and any $g \in F^{n}, d(g(x), x)$ is semicontinuous. Therefore, by Lemma 3.2 for each $n \geqslant 1$, $h_{n}(x)=\inf _{g \in F^{n}} d(g(x), x)=\inf \left\{d(g(x), x) \mid g \in F^{n}\right\}$ is semicontinuous. Thus, by Lemma 3.3, $\operatorname{Re}(G)=\tilde{F}^{-1}(0)$ is a dense $G_{\delta}$ - set in $X$. 
Lemma 3.6. If $(\mathrm{X}, \mathrm{G})$ is transitive, then $\operatorname{Re}(\mathrm{G} \times \mathrm{G})$ is a dense $\mathrm{G}_{\delta}$ of $\mathrm{X} \times \mathrm{X}$.

Proof. Let $\omega$ be a transitive point of $(X, G)$, then $\operatorname{orb}(\omega) \times \operatorname{orb}(\omega)$ is dense in $X \times X$. For any $(x, y) \in$ $\operatorname{orb}(\omega) \times \operatorname{orb}(\omega)$, we have $(x, y) \in \operatorname{Re}(G \times G)$. Hence, $\operatorname{Re}(G \times G)$ is dense in $X \times X$. By Lemma 3.5, $\operatorname{Re}(\mathrm{G} \times \mathrm{G})$ is a dense $\mathrm{G}_{\delta}$ set in $\mathrm{X} \times \mathrm{X}$.

Theorem 3.7. If $(X, G)$ is transitive and $\bigcap_{i=1}^{m} F i x\left(f_{i}\right) \neq \varnothing$, in which Fix(f) denotes the set of fixed points of $f$, then there exists a c dense Li-Yorke set of X. Specifically, $(X, G)$ is chaotic in the sense of Li-Yorke.

Proof. Set $\mathrm{D}=\operatorname{Re}(\mathrm{G} \times \mathrm{G}) \cap \mathrm{B}$, in which $\mathrm{B}$ is the same with the $\mathrm{B}$ in Lemma 3.4. Therefore, by Lemma 3.4 and Lemma 3.6, $\mathrm{D}$ is a residual set in $\mathrm{X} \times \mathrm{X}$. $\mathrm{X}$ is compact, then it is complete and separable. Then by [2] there exists a dense Mycielski set $K$ such that for any distinct $x_{1}, x_{2} \in K,\left(x_{1}, x_{2}\right) \in D$. Thus, $\left(x_{1}, x_{2}\right) \in$ $\operatorname{Re}(G \times G),\left(x_{1}, x_{2}\right) \in B$. By $\left(x_{1}, x_{2}\right) \in B$, for any $\delta>0$ there exists $g \in G$, such that $d\left(g\left(x_{1}\right), g\left(x_{2}\right)\right)<\delta$. By $\left(x_{1}, x_{2}\right) \in \operatorname{Re}(G \times G)$, there exist $\left\{g_{n_{i}}\right\}_{i=1}^{\infty}, g_{n_{i}} \in F^{n_{i}}, i \geqslant 1$ such that

$$
\lim _{i \rightarrow \infty}\left(g_{n_{i}} \times g_{n_{i}}\right)\left(x_{1}, x_{2}\right)=\left(x_{1}, x_{2}\right) .
$$

Hence there exists $\varepsilon_{0}=\frac{d\left(x_{1}, x_{2}\right)}{2}>0$, such that there are infinitely many $g \in G$ satisfying $d(g(x), g(y))>\varepsilon_{0}$. So for any distinct $x_{1}, x_{2} \in K,\left(x_{1}, x_{2}\right)$ is a Li-Yorke pair. Then, $K$ satisfies the condition of the present theorem.

Corollary 3.8. If $(\mathrm{X}, \mathrm{G})$ is transitive and $\mathrm{P}\left(\mathrm{f}_{1}\right) \cap \mathrm{P}\left(\mathrm{f}_{2}\right) \neq \varnothing$, then there exists a c dense Li-Yorke set of $\mathrm{X}$. Specifically, $(\mathrm{X}, \mathrm{G})$ is chaotic in the sense of Li-Yorke.

Proof. Set $v=f_{1}^{n_{1}}(v)=f_{2}^{n_{1}}(v)$, in which $n_{1}, n_{2}$ are positive integers, then $f_{1}^{n_{1} n_{2}}(v)=f_{2}^{n_{1} n_{2}}(v)=v$. Let $h_{i}=f_{i}^{n_{1} n_{2}}, i=1,2$, then $v \in \operatorname{Fix}\left(f_{1}\right) \bigcap \operatorname{Fix}\left(f_{2}\right)$. Denote the Abelian group generated by $h_{1}, h_{2}$ by $H$, then $\mathrm{H} \subset \mathrm{G}$.

Let $\omega$ be a transitive point of $(X, G)$. Set $X_{0}=\overline{\operatorname{orb}(\omega, H)}=\overline{\{h(\omega) \mid h \in H\}}$, then $\omega$ is a transitive point of $\left(X_{0}, H\right)$. And it is easy to see $\left(X_{0}, H\right)$ is transitive. Hence by Theorem $3.7\left(X_{0}, H\right)$ is chaotic in the sense of Li-Yorke. Then $(X, G)$ is chaotic in the sense of Li-Yorke.

Next, we present the second main result. Firstly, we list a lemma and several propositions.

Lemma 3.9. If both $\left\{p_{i}\right\}$ and $\left\{q_{i}\right\}$ are the infinite increasing subsequences of positive integers sequence $\left\{m_{i}\right\}$, there exists an infinite increasing subsequence $\left\{\mathrm{t}_{j}\right\}$ of $\left\{\mathrm{m}_{\mathrm{i}}\right\}$ such that

$$
\operatorname{AR}\left(F,\left\{p_{i}\right\}\right) \cap \operatorname{DR}\left(F,\left\{q_{i}\right\}\right) \subset \operatorname{DCR}\left(F,\left\{t_{j}\right\}\right) .
$$

Proof. If $\left\{\mathrm{p}_{\boldsymbol{i}}\right\} \bigcap\left\{\mathrm{q}_{\boldsymbol{i}}\right\}=\left\{\mathrm{r}_{\mathbf{j}}\right\}$ is an infinite sequence,

$$
\operatorname{AR}\left(F,\left\{p_{i}\right\}\right) \cap \operatorname{DR}\left(F,\left\{q_{i}\right\}\right) \subset A R\left(F,\left\{r_{j}\right\}\right) \cap D R\left(F,\left\{r_{j}\right\}\right)=\emptyset .
$$

Then, the conclusion is obviously true.

If $\left\{p_{i}\right\} \bigcap\left\{q_{i}\right\}$ is a finite set, without loss of generality, let $\left\{p_{i}\right\} \bigcap\left\{q_{i}\right\}=\emptyset$. Let $(x, y) \in A R\left(F,\left\{p_{i}\right\}\right) \cap$ $\operatorname{DR}\left(F,\left\{q_{i}\right\}\right)$. Then, select an infinite increasing subsequence $\left\{t_{j}\right\}$ of $m_{i}$ such that for any $i \in N$,

$$
\left\{\mathrm{t}_{\mathrm{j}} \mid \mathrm{n}_{\mathrm{k}_{\mathrm{i}}-1}<\mathrm{j} \leqslant \mathrm{n}_{\mathrm{k}_{\mathrm{i}}}\right\} \subset\left\{\mathrm{p}_{\mathrm{i}}\right\}, \quad\left\{\mathrm{t}_{\mathrm{j}} \mid \mathrm{n}_{\mathrm{k}_{\mathrm{i}}}<\mathrm{j} \leqslant \mathrm{n}_{\mathrm{k}_{\mathrm{i}}+1}\right\} \subset\left\{\mathrm{q}_{\mathrm{i}}\right\} .
$$

Hence, for any $\varepsilon>0$, for large enough $i$, as $n_{k_{i}-1}<j \leqslant n_{k_{i}}$, there are at most finitely many elements $g \in \bigcup_{n_{k_{i}-1}<j \leqslant n_{k_{i}}} F^{t_{j}}$ such that $d(g(x), g(y)) \geqslant \varepsilon$. Let it be $M$. Thus,

$$
1 \geqslant \frac{\sum_{j=1}^{n_{k_{i}}} \sum_{\tau^{\left(t_{j}\right)} \in T^{\left(t_{j}\right)}} \chi_{[0, \varepsilon)}\left(d\left(g_{\tau^{\left(t_{j}\right)}}(x), g_{\left.\tau^{\left(t_{j}\right.}\right)}(y)\right)\right)}{\sum_{j=1}^{n_{k_{i}}} m^{t_{j}}} \geqslant \frac{\sum_{j=n_{k_{i}-1}+1}^{n_{k_{i}}} m^{t_{j}}-M}{\sum_{j=1}^{n_{k_{i}}} m^{t_{j}}}=1-\frac{\sum_{j=1}^{n_{k_{i}-1}} m^{t_{j}}+M}{\sum_{j=1}^{n_{k_{i}}} m^{t_{j}}} .
$$


Furthermore, as $i \rightarrow \infty$,

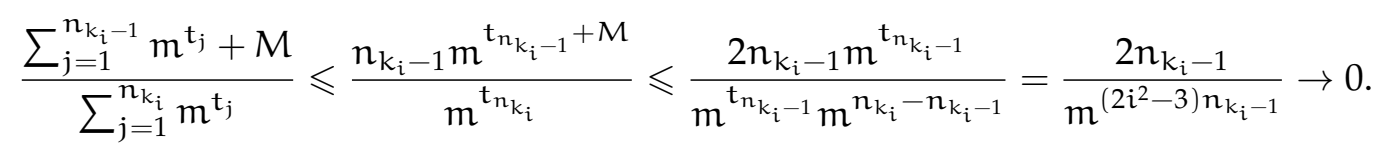

Thus, for any $\varepsilon>0, \limsup _{n \rightarrow \infty} \frac{\sum_{i=1}^{n} \sum_{\left.\tau_{i}\right)_{\in T} \sum_{T}^{\left(p_{i}\right)}} x_{[0, \varepsilon)}\left(d\left(g_{\left.\tau^{\left(p_{i}\right.}\right)}(x), g_{\tau}\left(\mathfrak{p}_{i}\right)(y)\right)\right)}{\sum_{i=1}^{n} m^{p_{i}}}=1$.

On the other hand, as $n_{k_{i}}<k \leqslant n_{k_{i}+1}$, there exists $\delta>0$ such that for any $g \in \bigcup_{j=n_{k_{i}+1}}^{n_{k_{i}+1}} F^{t_{j}}$, $d(g(x), g(y))>\delta$, therefore as $i \rightarrow \infty$,

$$
\begin{aligned}
& \frac{\sum_{j=1}^{n_{k_{i}+1}} \sum_{\tau^{\left(t_{j}\right)} \in T^{\left(t_{j}\right)}} \chi_{[0, \delta)}\left(d\left(g_{\tau^{\left(t_{j}\right)}}(x), g_{\tau^{\left(t_{j}\right)}}(y)\right)\right)}{\sum_{j=1}^{n_{k_{i}+1}} m^{t_{j}}} \\
& \leqslant \frac{\sum_{j=1}^{n_{k_{i}}} m^{t_{j}}}{\sum_{j=1}^{n_{k_{i}+1}} m^{t_{j}}} \leqslant \frac{n_{k_{i}} m^{t_{n_{k_{i}}}}}{m^{t_{n_{k_{i}+1}}}} \\
& \leqslant \frac{n_{k_{i}} m^{t_{n_{k_{i}}}}}{m^{t_{n_{k_{i}}} m^{2 k_{i} n_{k_{i}}-n_{k_{i}}}}}=\frac{n_{k_{i}}}{m^{\left(2 i^{2}-1\right) n_{k_{i}}}} \rightarrow 0 .
\end{aligned}
$$

Thus, there exists $\delta>0$ such that

$$
\liminf _{n \rightarrow \infty} \frac{\sum_{i=1}^{n} \sum_{\tau^{\left(\mathfrak{p}_{i}\right)} \in T^{\left(\mathfrak{p}_{i}\right)}} \chi_{[0, \delta)}\left(d\left(g_{\tau^{\left(p_{i}\right)}}(x), g_{\tau^{\left(p_{i}\right)}}(y)\right)\right)}{\sum_{i=1}^{n} m^{p_{i}}}=0 .
$$

Consequently, $\operatorname{AR}\left(F,\left\{p_{i}\right\}\right) \cap \operatorname{DR}\left(F,\left\{q_{i}\right\}\right) \subset \operatorname{DCR}\left(F,\left\{t_{j}\right\}\right)$.

Theorem 3.10. Let $\left\{n_{k}\right\}_{k=1}^{\infty}$ be a sequence of positive integers. Let both $\left\{A_{i}\right\}_{i=0}^{\infty}$ and $\left\{B_{i}\right\}_{i=0}^{\infty}$ be decreasing sequences of compact sets satisfying

$$
\bigcap_{i=0}^{\infty} A_{i}=\{a\}, \bigcap_{i=0}^{\infty} B_{i}=\{b\} .
$$

Put $\mathcal{C}=\left\{c=C_{0} C_{1} \cdots \mid C_{k} \in\left\{A_{k}, B_{k}\right\}, k=0,1, \cdots\right\}$. If for any sequence $c=C_{0} C_{1} \cdots$, there exists $x_{c} \in C_{0}$ such that $\mathrm{F}^{\mathrm{n}_{\mathrm{k}}}\left(\mathrm{x}_{\mathrm{c}}\right) \subset \mathrm{C}_{\mathrm{k}}$ for each $\mathrm{k} \geqslant 0$, then $(\mathrm{X}, \mathrm{G})$ is uniformly distributionally chaotic along a sequence. Furthermore, it is chaotic in the strong sense of Li-Yorke.

Proof. Let $\mathrm{E} \subset \Sigma_{2}$ be an uncountable set such that for any distinct points $s=s_{0} s_{1} \cdots, t=t_{0} t_{1} \cdots \in E, s_{n}=$ $t_{n}$ for infinitely many $n$ and $s_{m} \neq t_{m}$ for infinitely many $m$. For each $s=s_{0} s_{1} \cdots \in E$, by hypothesis, we can choose $x_{s} \in X$ such that for $i=0,1$,

$$
F^{p_{i}}\left(x_{s}\right) \subset \begin{cases}A_{i} & s_{i}=0 \\ B_{i} & s_{i}=1,\end{cases}
$$

and for any $n \geqslant 1, n !<k \leqslant(n+1) !$

$$
F^{p_{k}}\left(x_{s}\right) \subset \begin{cases}A_{k} & s_{n}=0 \\ B_{k} & s_{n}=1\end{cases}
$$

Put $D=\left\{x_{s} \mid s \in E\right\}$. Clearly, if $s \neq t$, then $x_{s} \neq x_{t}$. Because $E$ is uncountable, $D$ is uncountable. Let $x_{s}, x_{t} \in D$ be any different points, where $s=s_{0} s_{1} \cdots, t=t_{0} t_{1} \cdots \in E$. By the property of $E$, there exist sequences of positive integers $n_{i} \rightarrow \infty$ and $m_{i} \rightarrow \infty$ such that $s_{n_{i}}=t_{n_{i}}$ and $s_{m_{i}} \neq t_{m_{i}}$ for all $i$. 
Firstly, for any given $\delta>0$, we have so large $n_{I_{1}}$ that as $n_{i}>n_{I_{1}}$, for any $g \in F^{l_{n_{i} !+1}}, d(g(x), g(y))<\delta$. Put $p_{i}=l_{n_{i} !+1}, i=0,1, \cdots$, then $D \subset A R\left(F,\left\{p_{i}\right\}\right)$.

Secondly, let $\varepsilon=\frac{d(a, b)}{2}>0$. For $m_{I_{2}}$ large enough, as $m_{i} \geqslant m_{I_{2}}$, for any $g \in F^{l_{m_{i} !+1}}$, we have $d(g(x), g(y))>\varepsilon$. Put $q_{i}=l_{m_{i+I_{2}} !+1}, i=0,1, \cdots$, then $D \subset D R\left(F,\left\{q_{i}\right\}\right)$.

Consequently, $\mathrm{D} \subset \mathrm{AR}\left(\mathrm{F},\left\{\mathrm{p}_{i}\right\}\right) \cap \mathrm{DR}\left(\mathrm{F},\left\{\mathrm{q}_{i}\right\}\right)$. By Lemma 3.9 and the above proof, $(\mathrm{X}, \mathrm{G})$ is uniformly distributionally chaotic along a sequence. Furthermore, By Proposition 2.10 it is in the strong sense of Li-Yorke chaotic.

Example 3.11. Suppose that $\left(\Sigma_{2}, \sigma\right)$ is the one-sided symbolic dynamical system. Let the multiple mappings $F=\left\{\sigma, \sigma^{2}\right\}$ on $\Sigma_{2}$ and $G=\bigcup_{n=1}^{\infty} F^{n}$, then

(1) $\left(\Sigma_{2}, G\right)$ is sequentially distributionally chaotic and it is uniform.

(2) $\left(\Sigma_{2}, G\right)$ is chaotic in the strong sense of Li-Yorke.

Proof. Let $A_{i}=\left\{x=x_{0} x_{1} \cdots \mid x_{j}=0,0 \leqslant j \leqslant i\right\}, B_{i}=\left\{x=x_{0} x_{1} \cdots \mid x_{j}=1,0 \leqslant j \leqslant i\right\}$, then both $\left\{A_{i}\right\}_{i=0}^{\infty}$ and $\left\{B_{i}\right\}_{i=0}^{\infty}$ are decreasing sequences of compact sets satisfying

$$
\bigcap_{i=0}^{\infty} A_{i}=\{000 \cdots\}, \quad \bigcap_{i=0}^{\infty} B_{i}=\{111 \cdots\} .
$$

Let $\left\{p_{k}\right\}_{k=0}^{\infty}$ satisfy $p_{0}=1$ and $p_{k+1}=2 p_{k}+k+1$ for each $k \geqslant 0$. Then, for any $c=C_{0} C_{1} \cdots$, there exits

$$
x_{c}=t_{0}^{p_{0}} t_{0}^{p_{0}} t_{0}^{0+1} t_{1}^{p_{1}} t_{1}^{1+1} t_{2}^{p_{2}} t_{2}^{2+1} \cdots t_{k}^{p_{k}} t_{k}^{k+1} t_{k+1}^{p_{k+1}} t_{k+1}^{k+1+1} \cdots \text {, }
$$

where for $k \geqslant 0$

$$
t_{k}= \begin{cases}0 & C_{k}=A_{k} \\ 1 & C_{k}=B_{k}\end{cases}
$$

and $t^{n}$ denotes a finite sequence containing only symbol $t$ with the length of $n$, satisfying $x_{c} \in C_{0}$ and $F^{p_{k}}\left(x_{c}\right) \subset C_{k}$ for each $k \geqslant 0$.

Thus, by Theorem 3.10, we have $\left(\Sigma_{2}, \mathrm{G}\right)$ is uniformly distributionally chaotic along a sequence and chaotic in the strong sense of Li-Yorke.

\section{Acknowledgment}

This work is supported by the Independent Research Foundation of The Central Universities No. DC 201502050201, the Autonomous Research Foundation for colleges and universities under the Party Central Committee (DC 201502050410) and the NSFC (No. 11271061).

\section{References}

[1] G. Cairns, A. Kolganova, A. Nielsen, Topological transitivity and mixing notions for group actions, Rocky Mountain J. Math., 37 (2007), 371-397. 1

[2] E. Kontorovich, M. Megrelishvili, A note on sensitivity of semigroup actions, Semigroup Forum, 76 (2008), $133-141$. 1,3

[3] F. Polo, Sensitive dependence on initial conditions and chaotic group actions, Proc. Amer. Math. Soc., 138 (2010), $2815-$ 2826. 1

[4] O. V. Rybak, Li-Yorke sensitivity for semigroup actions, Ukrainian Math. J., 65 (2013), 752-759. 3.1

[5] L.-D. Wang, Y.-N. Li, Y.-L. Gao, H. Liu, Distributional chaos of time-varying discrete dynamical systems, Ann. Polon. Math., 107 (2013), 49-57. 1

[6] L.-D. Wang, G.-F. Liao, S.-M. Huan, Distributional chaos in a sequence, Nonlinear Anal., 67 (2007), 2131-2136. 1

[7] X.-X. Wu, Chaos of transformations induced onto the space of probability measures, Internat. J. Bifur. Chaos Appl. Sci. Engrg., 26 (2016), 12 pages. 1 
[8] X.-X. Wu, A remark on topological sequence entropy, Internat. J. Bifur. Chaos Appl. Sci. Engrg., 27 (2017), 7 pages.

[9] X.-X. Wu, G.-R. Chen, Sensitivity and transitivity of fuzzified dynamical systems, Inform. Sci., 396 (2017), 14-23.

[10] X.-X. Wu, P. Oprocha, G.-R. Chen, On various definitions of shadowing with average error in tracing, Nonlinearity, 29 (2016), 1942-1972.

[11] X.-X. Wu, X. Wang, On the iteration properties of large deviations theorem, Internat. J. Bifur. Chaos Appl. Sci. Engrg., 26 (2016), 6 pages.

[12] X.-X. Wu, J.-J. Wang, G.-R. Chen, $\mathscr{F}$-sensitivity and multi-sensitivity of hyperspatial dynamical systems, J. Math. Anal. Appl., 429 (2015), 16-26.

[13] X.-X. Wu, X. Wang, G.-R. Chen, On the large deviations of weaker types, Internat. J. Bifur. Chaos Appl. Sci. Engrg., 27 (2017), 12 pages.

[14] X.-X. Wu, L.-D. Wang, G.-R. Chen, Weighted backward shift operators with invariant distributionally scrambled subsets, Ann. Funct. Anal., 8 (2017), 199-210.

[15] X.-X. Wu, L.-D. Wang, J.-H. Liang, The chain properties and average shadowing property of iterated function systems, Qual. Theory Dyn. Syst., 2016 (2016), 9 pages.

[16] X.-X. Wu, L.-D. Wang, J.-H. Liang, The chain properties and Li-Yorke sensitivity of zadehs extension on the space of upper semi-continuous fuzzy sets, Iran. J. Fuzzy Syst., (Accepted). 1

[17] J.-C. Xiong, Chaos in a topologically transitive system, Sci. China Ser. A, 48 (2005), 929-939. 1, $3.2,3.3$ 